\title{
On Translation of Verses in Sanyan from the Perspective of Translation as Adaptation and Selection
}

\author{
Lin Tian \\ School of Foreign Languages, Sichuan University of Arts and Science, Dazhou, China \\ Email: tianlin1984@126.com
}

How to cite this paper: Tian, L. (2018) On Translation of Verses in Sanyan from the Perspective of Translation as Adaptation and Selection. Open Journal of Social Sciences, 6, 190-201. https://doi.org/10.4236/jss.2018.68015

Received: July 25, 2018

Accepted: August 21, 2018

Published: August 24, 2018

Copyright $\odot 2018$ by author and Scientific Research Publishing Inc. This work is licensed under the Creative Commons Attribution International License (CC BY 4.0).

http://creativecommons.org/licenses/by/4.0/

\section{(c) (i) Open Access}

\begin{abstract}
Verse, frequently seen in Chinese literary works, is a significant component of huaben novels. As a representative masterpiece of vernacular short stories, Sanyan is no exception, and many rhythmical verses have been used and become a prominent feature. The present paper studies the translation of verses in Sanyan by Shuhui Yang and Yunqin Yang from the perspective of Translation as Adaptation and Selection. The study finds that the Yang have made active adaptation to the textual and non-textual eco-environment, which makes it possible for this translational activity to take place. The two translators have also achieved three-dimensional transformation by making adaptive selections. Since the translational eco-environment is dynamic, the strategies or skills adopted by the translators are flexible. All in all, the Yang, while guaranteeing the semantic loyalty to the source verse, tries to reproduce the form and deliver the cultures by adding endnotes so as to realize the communicative purpose.
\end{abstract}

\section{Keywords}

Translation, Verses, Sanyan, Translation as Adaptation and Selection

\section{Introduction}

Rhythmical verses have always been used in Chinese literature due to literary traditions, especially in huaben novels which refer to the vernacular short stories with story-telling genre. With specific functions, verse is regarded as a significant part of huaben novels. Because Chinese and English fall into the category of different family languages embedded in distinctive cultures, the translation of verses can be extremely difficult. "Jakobson even holds that the verse is untran- 
slatable whether it is intralingual or interlingual, and the verse translation can only be achieved through creative transplantation.” ([1], p. 256). There are a large number of rhythmical verses in Sanyan, all of which have been translated by Shuhui Yang and Yunqin Yang (hereinafter "the Yang") in their translated works, Stories Old and New, Stories to Caution the World and Stories to Awaken the World. This translation of Sanyan is the most complete translation among all the existing translated versions, and has been collected into the $\mathrm{Li}$ brary of Chinese Classics. Therefore, it deserves our exploration as to how the verses are translated by the Yang.

The previous studies on the verse translation in Sanyan are quite limited. For instance, Sun Buzhong [2] firstly traces the use of verses in huaben novels, studies their forms, functions and developing process, and then has a detailed analysis of the classical masterpiece of each stage of development. Though he has studied the verses and the representative vernacular story in each period, but only a small proportion of his doctoral thesis has touched upon Sanyan. Some scholars focus their study on the verses in Sanyan, such as the study on opening poems [3], or the roles of verses and their aesthetic values [4]. As for the studies on the translation of verses, Zhuang Qunying and Li Tingxin [5] study the translated opening poems in Selected Chinese Stories of the Song and Ming Dynasties, a selected translation of Sanyan by Yang Xianyi and Gladys Yang, finding that their translated poems can meet the criteria raised by Liu Zhongde and $\mathrm{Xu}$ Yuanchong.

According to the present author's knowledge, little research on the Yang's translated verses has been done. Translation as Adaptation and Selection, a theory developed based on ecology can provide the research a relatively new angle. Therefore, the study on the Yang's translation of verses from the perspective of Translation as Adaptation and Selection can not only enrich the translation study of Sanyan, but also explore the feasibility of this theory to analyze the translation of verses.

\section{The Adoption of Verses in Sanyan}

The adoption of verses is one of the dominating feature in huaben novels. Verses in rhyme are used to enhance the artistic power of the works, to promote the status of huaben novels by story-tellers and literary men, and to make the vernacular novels more alive by ancient Chinese writers ([2] p. 1). huaben, originally the script for professional story-tellers, is a literature form popular due to the development of folk story-telling art. "The storyteller-narrator asks questions of his simulated audience, converses with them, makes explicit references to his own stories, and intersperses his narrative with verses and poems". ([6], p. 21). Since the vernacular stories derive from the story-telling technique, the combination of both proses and verses, or narration with verses and poems has become the unique artistic form for huaben novels from the very beginning.

In Sanyan, the linguistic style is also the combination of proses and verses, 
and rhythmical verses can be seen in almost every part of each story. "There are lots of verses in the Sanyan collections-599 verses in Stories Old and New, 708 verses in Stories to Caution the World and 657 verses in Stories to Awaken the World." ([7], p. 11). There are an opening poem and an ending poem in each story of the collections, and rhythmical verses also appear in the main body of the story. Some cues are usually used before the verses, indicating the appearance of rhythmical verses, such as "there is a poem in testimony" (youshi weizheng), "truly" (zhengshi), "there is a poem that says" (shiyue/shiyun) ([8], p. 6). The rhythmical verses in Sanyan are used to describe settings, situations, sceneries, characters, etc., and most are delivered in the form of story-telling. Besides, the rhythmical verses embedded also "perform the educating function of saving people from degeneration" ([2], pp. 3-4). Specifically speaking, the opening poems or verses in Sanyan are meant to tell the readers the theme of a story, or create certain images and lighten atmosphere, or express emotions by telling stories which are similar or contrary to the main story, while the ending poems or verses aim to summarize the story, or educate the audience by advising them to learn a lesson ([8], p. 2). The verses in the main body of the story are adopted to describe the characters, the setting or the environment, or to comment on the narrated event ([5], p. 147).

\section{General Introduction to Translation as Adaptation and Selection}

Translation as Adaptation and Selection is believed to be the theoretical basis of Eco-translatology, which is put forward by Professor Hu Gengshen. According to the theory, translation is the cyclical alternations of the translator's adaptation and selection. To be specific, translation process involves the translator's adaptation to the translational eco-environment which takes the source text as its typical important component, and the selection to the target text of the translational eco-environment which takes the translator as its typical important component ([9], p. 1). As we can see translational eco-environment is one of the key terms in this theory. It refers to the aggregation of the relevant text, cultural context, spiritual and material stuff as well as "translation community" which indicates all the parties involved in the translational activity including the translator, the readers, the writer, the sponsor, the publisher and the commenters ([10], p. 7).

The translation principle, within the framework of this theory, can be summarized as multi-dimensional adaptation and adaptive selection, which means that the translator should achieve multi-dimensional adaptation to the translational eco-environment, and then make corresponding adaptive selections. The translation method is briefly summarized as "the three-dimensional transformation", namely, adaptive transformation from the linguistic, cultural and communicative dimension. Adaptive transformation from the linguistic dimension indicates the translator lays emphasis on the transformation of source text's linguistic form after actively adapting to the eco-environment. As for adaptive transfor- 
mation from the cultural dimension, the translator stresses on the cultural differences and cultural transmission between the source culture and the target culture. The translator should not distort the cultural elements expressed in the source text simply based on the target culture, but to take the whole source cultural environment into consideration ([9], p. 2). With regard to adaptive transformation from the communicative dimension, the translator should emphasize the communicative purpose reflected in the source text, and try to achieve the communicative function in his translation ([10], p. 8).

There is no denying that multi-dimensional adaptation and adaptive selection can provide us with new angles to study the Yang' translation, and the threedimensional adaptive transformation can offer us different dimensions to see the Yang's translation of verses. Therefore, taking this theory as the basis, the present paper mainly emphasizes two aspects in the following two parts: The Yang's adaptation to the translational eco-environment and the Yang's adaptive selections in the translating process of verses in Sanyan.

\section{The Yang's Adaptation to the Translational Eco-Environment}

The Yang choose Feng Menglong's Sanyan as the source text, and finish this complete translated version after years of hard work. This just reflects the translators' adaptation to the translational eco-environment, which not only includes the source textual environment but also the non-textual environment. As discussed in the previous part, source text is one of the components in the translational eco-environment, and it itself can be also seen as a microenvironment, which is named as "textual environment" in this part. Apart from the source text, the rest components in the translational eco-environment just fall to the realm of "non-textual environment".

\subsection{The Yang's Adaptation to the Non-Textual Environment}

Translator's adaptation to fit the translational eco-environment makes it possible for the translational activity. "The translator must adapt to the translational eco-environment in order to be able to select the specific target texts." ([11], p. 284). The non-textual eco-environment involves the source culture and the target culture.

In China, the translation of classical masterpiece is greatly promoted. Especially, the Library of Chinese Classics in a Chinese-English format have been published, which strongly stimulates the translators' interest in engaging in translational activities. Besides, many scholars overseas focus their study on vernacular stories in China including Sanyan, and an increasing number of readers develop their interest in China and Chinese culture. However, the translated versions before the Yang's are only translations with selected materials. As is mentioned by the Yang, "none of the three Sanyan collections had been translated in its entirety before the end of the $20^{\text {th }}$ century." ([6], p. 27). For most of 
the translated versions, "the storyteller's rhetoric, the verses, and the prologue stories were often deleted", and "the interlineal and marginal comments, generally believed to have been made by Feng Menglong himself, were omitted" ([6], pp. 27-28). Therefore, it is of great need to produce a complete translation of Sanyan.

\subsection{The Yang's Adaptation to the Textual Environment}

“The source text has pride of place as it 'selects' the translator in the eco-environment. But this reversible selection could also be regarded as the translator's adaptation to the source text." ([11], p. 285). The Yang's personal experience and research interest can also be regarded as their active adaptation to the translational eco-environment.

The Chinese American scholar Shuhui Yang, professor of Chinese at Bates College, graduated from Fudan University in 1982. In 1984, he went to the United States for further education under the supervision of Robert E. Hegel, a renowned sinologist, and received his $\mathrm{Ph} . \mathrm{D}$ in Chinese and Comparative Literature from Washington University in St. Louis. ([12], p. 75). In his doctoral thesis Storytelling and Ventriloquism: The Voice of a Literatus in the Sanyan Collections, he compared Feng Menglong with Daniel Defoe, the great British novelist. His first book Appropriation and Representation: Feng Menglong and the Chinese Vernacular Story is by far the only book about Feng Menglong and the Sanyan Collections written in English. Yunqin Yang, the co-translator and also Shuhui Yang's wife, used to work in Shanghai Translation Publishing House, and now work in UN headquarter as a simultaneous interpreter. For forty years, the two translators have developed a habit that whenever a delicate expression is encountered in their reading, it will be noted down on a card. All the cards that are collected have become a "database", where they can check during their translation of Sanyan ([13], p. 101).

From the above, it can be seen that the Yang have made selective adaptations to the eco-environment, not only the textual but also the non-textual environment. The social and educational needs as well as personal professional interest have made the Yang selected as the translators by the eco-environment. Meanwhile, this process reflects the Yang's active adaptation to the translational eco-environment, which provides quality guarantee for the translational activity.

\section{The Yang's Adaptive Selections in Translating Verses in Sanyan}

As is mentioned in the previous part, the translators have to make adaptive selections after adapting to the translational eco-environment. In the stage of adaptive selection, "the translator is the central figure, as he or she 'selects' and makes decisions about the form of the final target text in the translational eco-environment. In other words, the translator is active in carrying out a selection leading to the production of a translation." ([11], p. 285). A successful 
translation is believed to have achieved the adaptive transformation from the linguistic, cultural and communicative dimension, during which the concrete skills can be flexibly adopted based on different circumstances.

\subsection{Linguistic Dimensional Transformation}

The Yang have conducted the transformation from the linguistic dimension in translating the verses in Sanyan, which is mainly discussed from the following two aspects, the choice of words and sentences. The following verses are given to illustrate the linguistic transformation of the Yang.

Verse 1:

儿孙自有儿孙福, 莫与儿孙作马牛。

—ST by Feng ([6], p. 50)

Your children will have their own share of fortune;

Don't serve them meekly like beasts of burden.

- TT by the Yang ([6], p. 51)

Verse 2:

不是冤家不聚头, 冤家相聚几时休?

早知死后无情义, 索把生前恩爱勾。

—ST by Feng ([6], p. 58)

If not so fated, lovers will never meet;

But once they do, when will their cursed love end?

Had you known that no love lasts beyond the grave,

You would have cut off all love before your death.

- TT by the Yang ([6], p. 59)

In verse 1, “福” and “马牛” are respectively translated by the Yang into “share of fortune" and "beasts of burden", which are not only similar in form but also delicately express the connotation of the words in the source text. "Beasts of burden", originally referring to those animals which are trained to provide tractive force for human beings, is used here to signify those parents and grandparents who sacrifice a lot for the happiness of the younger generations. Thus, the choice of words is appropriate. In verse 2, “冤家” is translated into “lover”, and "cursed love" is used to indicate the love between the lovers. While the Yang translate “死后”, they use "beyond the grave" instead of “after death". The former choice "grave" can effectively create a much more vivid image than the word "death" for the target readers.

Beside the word level, the Yang have also made adaptive selections on the sentence level. In the above two examples, more pronouns and conjunctions are used in the translation than in the source verses. In verse 1, “儿孙” appears three times in the source verse, while "children" is only used once, and pronouns are used to replace the word instead. In verse 2, the logical links, which are implied in the source verse, are added by the Yang, such as "if" and "but". Division skill is also used to translate the first two sentences, such as “不是冤家不聚头”, which is translated into "If not so fated, lovers will never meet". In addition, 
grammatical inversion is also adopted, such as "had you known that". All these transformations are natural and confirm to the target language habit, because Chinese lays emphasis on parataxis with bamboo-like structures, while English lays stress on hypotaxis with tree-like structures.

All in all, all these linguistic transformations are made in order to make the translation easily fit in the target language eco-environment. Both dictions and sentence patterns are well selected in the translation, which are not only semantically loyal to the original information, but also concise in style like the blank verses in the English language environment.

\subsection{Cultural Dimensional Transformation}

Translation of literary works not only involves the transformation from the linguistic aspect, but also the transformation of different cultures embedded in the language. "The cultural unequivalence has increased the difficulty of literary translation. Verse, as a special type of literature, is even more complicated and difficult to translate than any other literary type." ([5], p. 147). Verse itself, consisting many different forms, is a kind of culture. Diezishi and huiwenshi are two distinctive forms of Chinese verses, which are occasionally used in Sanyan.

Verse 3:

别离时闻漏转

忆静

期归阻久伊思

静思伊久阻归期, 久阻归期忆别离。

忆别离时闻漏转, 时闻漏转静思伊。

—ST by Feng ([14], p. 137)

I've missed her for long barred from returning

Quietly

I recall

water-clock dripped away when parting

Quietly I've missed her for long;

For long barred from returning, I recall [ the] parting.

I recall $[$ the] parting when away dripped $[$ the] water-clock.

Away dripped [the] water-clock quietly, I've missed her for long.

-TT by the Yang ([15], p. 240)

huiwenshi, also named palindrome verse, is a unique type of Chinese classic verses, which usually can be read vertically, horizontally, diagonally, in reverse or in a circle just due to the versatility of the Chinese written language. It has become popular since the Jin Dynasty and is adopted in many literary forms. Verse 3 is just a clockwise huiwenshi, expressing Qin Guan's miss to Su Xiaomei. In the original verse, each character in the clockwise verse appear twice in its interpretation verse right after it. Different from other type of verses, huiwenshi lays great stress on its form and appearance, so it becomes a crucial problem as to how to maintain this form in the translation so as to deliver to the readers its aesthetic beauty and cultural image of the verse. It can be seen that the Yang's 
translation, while achieving linguistic transformation, has also realized the cultural transformation by perfectly reproducing the form of this verse.

Verse 4:

寻寻受受, 冷冷清清, 凄凄惨惨戚戚。

乍暖还寒时候, 正难将息。

…..

梧桐更兼细雨, 到黄昏, 点点滴滴,

这次第, 怎一个愁字了得!

—ST by Feng ([14], p. 130)

Seeking, searching, in solitude and sorrow,

I find little peace in the capricious weather.

...

Amid the drip-drip of rain from the paulownia leaves,

My sorrow defies all descriptions by word.

Verse 4 is a verse with reiterative locution, which is calleddiezishi in Chinese. Characters of duplication are used in many ancient Chinese verses for artistic expressiveness. This verse excerpted from the Sanyan collections is written by $\mathrm{Li}$ Qingzhao and has been translated into many versions. In the first line of the source verse, seven pairs of reiterative locution are used to create a desolating atmosphere, including 寻(xun), 受 (mi), 冷(leng), 清(qing), 凄(qi), 惨(can) and 戚(qi). In the translation of this line, alliteration is adopted by the Yang to create the same atmosphere, which is more familiar to the target readers and thus can deeply touch them. This also shows the cultural phenomenon of reiterative locutions in succession is difficult to be reproduced in target language due to the linguistic differences. As for “点点滴滴”, the Yang try to use "drip-drip” to reproduce the same form of reiterative locution.

It can be derived from the above two cases that the Yang adopt different strategies when dealing with specific cultural phenomenon. In order to make the translation survive in the whole translational eco-environment, "the translator's selection of translation strategies, free or literal, domestication and foreignization, will depend on all the constant shifts and changes in the translational eco-environment." ([11], p. 286). In the translation of huiwenshi, the Yang have delicately reproduced the form without the sacrifice of the meaning. However, in the translation of this diezishi, the Yang first guarantee the delivery of meaning in a domesticated way, and meanwhile, try to present the cultural phenomenon of the source verse to the maximum extent.

\subsection{Communicative Dimensional Transformation}

The verses and rhymes created by the ancient litterateur contain classical allusions, which are usually expressed in the form of concise but connotation-profound words. To the native readers, these allusion-loaded words or expressions can easily remind them of the related cultures, but to the target readers from another culture, the literal translation of these words is just meaningless, 
and thus cannot be easily understood and accepted. There is no denying that this will lead to the breakdown of cross-cultural communication. As an ancient classical masterpiece, Sanyan contains many such allusions. The following example can display how they are transformed by the Yang from the dimension of communication.

Verse 5:

周郎妙计高天下，赔了夫人又折兵。

-ST by Feng ([16], p. 1094)

Zhou Yu's clever plan-how it was shattered!

He lost both-the lady and the battle.

-TT by the Yang ([16], p. 1095)

In verse 5, “周郎”, alias of Zhou $\mathrm{Yu}$, is a well-known historic figure in the source language eco-environment. Even without any further explanation, the source reader may get the deeper connotation implied as soon as they see the sentence. However, to target readers, they belong to a total different language eco-environment. Thus, in the communication process, the target readers cannot get the same effect simply through the literally translated works. Translator, as a key party in the translational eco-environment, can best make up for the loss of a specific allusion through certain translation strategies or skills. In this case, the Yang deliver the cultural allusion by adding endnotes at the end of the story, which goes as follows, "According to The Romance of the Three Kingdoms, Zhou Yu (175-210), military adviser to Sun Quan of Wu, devised a plan to capture Liu Bei, Sun's rival, by offering him Sun's sister as wife so as to lure him to the $\mathrm{Wu}$ region to pick up the bride. But Liu Bei's military adviser, the great strategist Zhuge Liang, saw through the plot and beat Zhou Yu at his own game by having Liu Bei successfully take away the bride and return to his own territory safe and sound. Zhou Yu led his troops in a chase but was defeated by Liu Bei's general Zhang Fei. This story is invariably cited in reference to situations whereby one ends up suffering a double loss through actions intended to produce a gain.” ([16], p. 1167). This endnote not only gives a detailed explanation about the story of Zhou $\mathrm{Yu}$, but also the implication of the verse. Many names of historical figures in the verses are given further explanation in the endnotes, so are the names of places. For instance,

Verse 6:

山外青山楼外楼, 西湖歌舞几时休?

暖风熏得游人醉, 只把杭州作泣州。

—ST by Feng ([17], p. 1312)

Hill beyond green hill, tower beyond tower,

When will songs and dances by West Lake ever cease?

Enchanted by the warm breezes,

The sightseers take Hangzhou for Bianzhou. ${ }^{1}$

-TT by the Yang ([17], p. 1313)

In verse 6 , the translation of the first two lines can offer the target readers a sharply contrasted image-the beautiful scenery and the never-ceasing songs 
and dances along the West Lake. Especially the use of interrogative sentence pattern and the word "ever" can let the readers feel the poet's hatred of the scene. However, in the last line of the source verse, two places are mentioned, namely, “杭州” and “泫州”, which are respectively transliterated into "Hangzhou" and "Bianzhou". To the target readers, they may have such puzzle, "Why do the sightseers take Hangzhou for Bianzhou?" If the cultural and historical background information cannot be delivered to the target readers, the communicative purpose can't be realized. Therefore, the Yang add an endnote, which is "Bianzhou, or Bianjing, present-day Kaifeng in Henan Province, was the capital of the Song dynasty before the court moved south to Hangzhou" ([17], p. 1399).

Actually, endnote is a translation technique that is favored by the Yang, since it can be seen from the translation of the Sanyan, many endnotes have been added at the end of each story, especially when there is a name of a place or a historic figure. All these aim to remove the barriers for readers, promote alien cultures and finally realize the communicative purpose. Therefore, the communicative dimensional transformation can be achieved with endnotes as one of the bridges between different sub eco-environments.

\section{Conclusion}

As Pound once said, "only when the translator can touch deep the soul of the writer of a verse, will he be able to produce a translation that echoes with the original verse in style and effect by traversing the cultural and linguistic barriers." ([1], p. 256). In the translation of verses in Sanyan, the Yang had given priority to the achievement of semantical loyalty to the source writer and text, but meanwhile, tried to deliver them with appropriate dictions and well-selected sentence patterns which can make the translation better survive in the translational eco-environment. With regard to cultural phenomenon such as huiwenshi and diezishi, the Yang just selected flexible strategies or skills based on concrete eco-environment, and did their utmost to reproduce the aesthetic beauty of the original form, on condition that the meaning carried by the source verse can be faithfully delivered to the target readers. Language, culture and communication can never be separated. Only when linguistic accuracy and cultural delivery are successfully achieved, will it be possible for the translation to perform its communicative function. Endnote is frequently adopted by the Yang to bridge the gap between different cultural eco-environment. As is mentioned by the Yang in their conference paper, they prefer to use endnote, simply because endnote doesn't appear on the same page with the source text, which allows the readers more freedom to choose their own way of reading, either neglecting the endnote for general readers, or studying the endnote for those readers such as scholars ([12], p. 77). This can be regarded as one of the Yang's adaptive selections after active adaptation to the translational eco-environment. Besides, the Yang's adaptation to the textual and non-textual eco-environment in the pre-interpreting process also matters a lot, which guarantees the adaptive transformation of the 
verses from the dimensions of linguistics, cultures and communications. Though detailed study of the Yang's verse translation has been carried out in the current paper, the qualitative approach is mainly adopted, and thus, the research findings may not be so systematic and comprehensive. Therefore, a thorough comparison between the verses of Sanyan and their corresponding translations is suggested for further study with quantitative approach.

\section{Acknowledgements}

This paper is sponsored by American Studies Center of Southwest Jiaotong University (Project Number: ARC2018024; Project Name: Study on American Heritage Education)

\section{Conflicts of Interest}

The authors declare no conflicts of interest regarding the publication of this paper.

\section{References}

[1] Fang, M.Z. (2011) A Dictionary of Translation Studies in China. Shanghai Foreign Language Education Press, Shanghai.

[2] Sun, B.Z. (2002) Study on Verses in Ancient Vernacular Stories. Ph.D. Thesis, Shanghai Normal University, Shanghai.

[3] Zhang, X.Q. (2011) On Opening Poems of Sanyan Erpai. Modern Chinese, 5, 20-21.

[4] Ren, X.Y. (2001) On the Poems in Sanyan. Journal of Huaiyin Teachers College (Social Science), 6, 763-765.

[5] Zhuang, Q.Y. and Li, X.T. (2010) On the Translation of Poetry in Yang's Selected Chinese Stories of the Song and Ming Dynasties-An Analysis of Translated Opening Poems. Journal of Yichun College, 7, 147-149, 175.

[6] Feng, M.L. (2009) Stories to Caution the World (Yang, S.H. and Yang, Y.Q., Trans. Vol. 1). Yuelu Publishing House, Changsha.

[7] Hao, Y. (2017) Comparison between Two Translations of Didactic Verses in the Sanyan Collections-From the Perspective of Functional Equivalence. Master's Thesis, Shanghai International Studies University, Shanghai.

[8] Tan, Y.J. (2005) Study on Languages in Sanyan Erpai. Bashu Publishing House, Sichuan.

[9] Hu, G.S. (2008) Viewing Translation Theory from Terminology. Shanghai Journal of Translators, 2, 1-5.

[10] Hu, G.S. (2011) Eco-Translatology: Research Foci and Theoretical Tenets. Chinese Translators Journal, 2, 5-9, 95.

[11] Hu, G.S. (2003) Translation as Adaptation and Selection. Perspectives. Studies in Translatology, 11, 283-291. https://doi.org/10.1080/0907676X.2003.9961481

[12] Yan, M. (2013) Both the Inner Beauty and the Rebuilding Ability-Introduction to the First Complete English Translation of "Sanyan". Chinese Translators Journal, 34, 75-79.

[13] Yang, S.H. and Yang, Y.Q. (2016) Exploring the "Shortcut" of Achieving Loyalty of Language Style-Taking English Translation of Sanyan as an Example. Chinese 
Translators Journal, 37, 101-105.

[14] Feng, M.L. (2002) Sanyan Erpai Xingshi Hengyan. Tianjin Ancient Books Publishing House, Tianjin.

[15] Feng, M.L. (2012) Stories to Awaken the World: A Ming Dynasty Collection. (Yang, S.H. and Yang, Y.Q., Trans, Vol. 3). University of Washington Press, Seattle.

[16] Feng, M.L. (2009) Stories to Caution the World (Yang, S.H. and Yang, Y.Q., Trans, Vol. 3). Yuelu Publishing House, Changsha.

[17] Feng, M.L. (2011) Stories to Awaken the World (Yang, S.H. and Yang, Y.Q., Trans, Vol. 3). Yuelu Publishing House, Changsha. 